\title{
Hybrid Differential Software Testing
}

\author{
Yannic Noller
}

\begin{abstract}
Differential software testing is important for software quality assurance as it aims to automatically generate test inputs that reveal behavioral differences in software. Detecting regression bugs in software evolution, analyzing side-channels in programs, maximizing the execution cost of a program over multiple executions, and evaluating the robustness of neural networks are instances of differential software analysis to generate diverging executions of program paths. The key challenge thereby is to simultaneously reason about multiple program paths, often across program variants, in an efficient way. Existing work in differential testing is often not (specifically) directed to reveal a different behavior or is limited to a subset of the search space. This work proposes the concept of Hybrid Differential Software Testing (HYDIFF) as a hybrid analysis technique to generate difference revealing inputs. HYDIFF consists of two components that operate in a parallel setup: (1) a search-based technique that inexpensively generates inputs and (2) a systematic exploration technique to also exercise deeper program behaviors. HYDIFF's search-based component uses differential fuzzing directed by differential heuristics. HYDIFF's systematic exploration component is based on differential dynamic symbolic execution that allows to incorporate concrete inputs in its analysis. HYDIFF is evaluated experimentally with applications specific for differential testing. The results show that HYDIFF is effective in all considered categories and outperforms its components in isolation.
\end{abstract}

Please note that this book chapter is a condensed version of the original dissertation [1] and the corresponding publications [2-7].

Y. Noller $(\bowtie)$

National University of Singapore, Singapore, Singapore

e-mail: yannic.noller@acm.org

M. Felderer et al. (eds.), Ernst Denert Award for Software Engineering 2020, https://doi.org/10.1007/978-3-030-83128-8_9 


\section{Introduction}

Software Engineering (SE) is the "systematic application of scientific and technological knowledge, methods, and experience to the design, implementation, testing, and documentation of software," as defined by the IEEE [8]. A key insight in the community is that "programming-in-the-large" [9] is much more complex than "programming-in-the-small", and hence, requires a proper engineering approach [10]. The objective of SE research is the study of how to develop software (in the large), in order to provide the scientific knowledge and methods to do so. It searches for techniques to improve, simplify, and support software development. A crucial part of software development is software quality assurance facilitated by software testing [8], as it searches for errors in software.

Therefore, software testing is an essential part of software development, which is widely applied by practitioners and is also the focus of numerous research projects [11]. The goal is to provide confidence in the correctness of software by searching for errors in its behavior. The manual creation of test cases can be very expensive and time consuming. Therefore, recent research has focused on automated test input generation [11, 12]. Identified errors can be investigated and fixed, and so, software testing contributes to the quality of the software.

A special area in this research field is differential software testing, which aims to identify behavioral differences in software, that is, differences in the execution behavior of a program. Such differences can be represented by several forms, for example, a difference in the direct output of a program, a difference in the execution time, or also a difference in the covered code fragments. Differential program analysis (here also called differential software testing) means the analysis of one or multiple programs in order to reveal behavioral differences. In general, the search for behavioral differences can be separated into two categories: It can reveal divergences between two execution paths (1) of different program versions or (2) within the same program. Figure 1 illustrates these two types of differential analysis. Category (1) (on the left side of Fig. 1) searches for input $\mathrm{x}$ that leads to a different execution behavior between program $\mathrm{P}$ and its successive variant $\mathrm{P}^{\prime}$. Note that generally $\mathrm{P}$ and $\mathrm{P}^{\prime}$ can be completely different programs. Category (2) (on the right side of Fig. 1) searches for two different inputs $x$ and $y$ that lead to a different execution behavior for (the same) program P. Depending on the application it can be interesting how similar the two inputs are. Both analysis categories require multiple program executions, which makes differential software testing a challenging problem.

For example, differential testing is often applied in software maintenance to perform regression testing [13, 14], where the goal is to reveal differences between two successive software versions. Such differences can be observed, for example, along the control-flow or in the actual output of the execution. Other flavors of differential program analysis are used in the area of software security to perform automated vulnerability detection, for example, with regard to worst-case execution paths [15, 16] or side channels [17-19]. For example, algorithmic complexity vulnerabilities can be exploited to cause a denial of service attack. Side-channel 


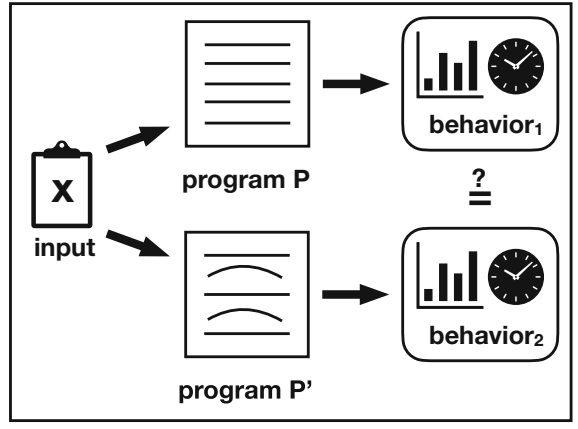

1

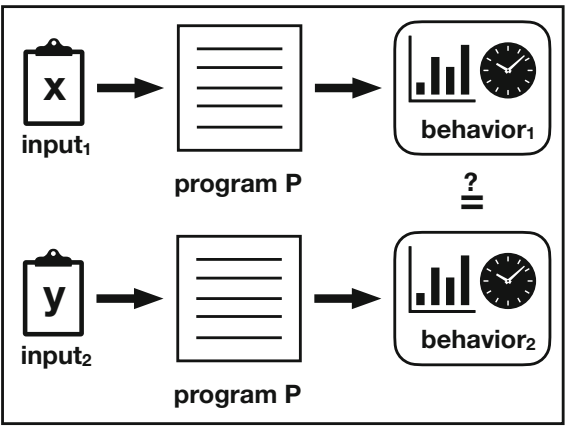

2

Fig. 1 Two categories of differential software testing

vulnerabilities can be exploited to reveal sensitive information by observing the nonfunctional characteristics of the program behavior, such as the execution time, memory consumption, response size, or network traffic. A novel application of differential analysis is the robustness analysis of neural networks [20,21], which concerns the software reliability. This kind of analysis aims to identify two inputs that differ only very slightly, that is, for a human almost imperceptible, but for which the neural network produces a different output (e.g., it results in different classifications) [22]. The differences between such inputs are also called adversarial perturbations and represent major safety and security issues.

This work focuses on the above problem of differential software testing with its numerous application areas. In particular, it develops concepts and techniques for the automated generation of test inputs that reveal behavioral differences in software. Furthermore, it provides a general framework, which can be applied to all the mentioned analysis types. In summary, differential software testing is investigated in the context of the following applications:

\section{Application Areas}

A1 Regression analysis - The search for behavioral differences with the same input in successive program versions.

A2 Worst-case complexity analysis-The search for worst-case triggering inputs that perform significantly different than the average case.

A3 Side-channel analysis - The search for side-channel vulnerabilities in security-critical applications, which involves analyzing correlations between resource usages over multiple program paths.

A4 Robustness analysis of neural networks - The search for adversarial behaviors in neural networks, which requires reasoning about multiple network executions. 
The existing techniques in differential testing come with their own disadvantages: Many of them are not directed to differential behavior, are not able to solve necessary constraints to reach deep program behavior, or rely on an exhaustive exploration. They are limited in the effectiveness of their analysis. An efficient and effective testing approach asks for a hybrid execution setup [16, 23]. Therefore, this work proposes the concept of Hybrid DIFFerential Software Testing (HyDIFF), which combines search-based testing with a systematic exploration technique. More specifically, this concept aims to combine the speed of search-based fuzzing [24] and the systematic exploration of symbolic execution in a parallel setup. Both components perform their own differential analysis, while they exchange (interesting) inputs to support each other. This provides a generally applicable, differential software testing approach, which in particular can be applied to the mentioned application scenarios. The evaluation of HYDIFF investigates whether it can reveal behavioral differences in software and how the hybrid combination performs in contrast to its components in isolation. HYDIFF is evaluated based on a quantitative analysis with benchmarks taken from the above-mentioned application scenarios.

\section{Core Contributions}

C1 The concept of differential fuzzing that incorporates various differential metrics to provide a general differential analysis. In particular it allows the search for side-channel vulnerabilities because it also uses cost metrics to determine the cost difference of two executions.

C2 The concept of differential dynamic symbolic execution as a technique to perform a dynamic symbolic exploration driven by differential heuristics, which allows to incorporate concrete inputs during the analysis. This allows the continuous guidance of the symbolic exploration to interesting program behaviors.

C3 The concept of a general hybrid approach in differential program analysis, which combines the strengths of single techniques in this research field. This concept closes a gap in the research of differential program analysis that is currently performed by specialized techniques with their own advantages and disadvantages. The hybrid concept allows to combine their advantages and compensate their disadvantages.

C4 The concept of a hybrid setup for applying fuzzing and symbolic execution in parallel as an alternative to already existing hybrid approaches in test input generation. The parallel environment allows that both techniques can continue their own powerful differential analysis while being supported by the results of the other component. 


\section{Hybrid Differential Testing: Assumptions and Concept}

Assumptions The overall goal of this work is to generate test inputs that expose behavioral differences. The assumption thereby is that existing tests, like a regression test suite, are not sufficient to expose the behavioral differences. This assumption is valid because existing work has shown that regression test suites need augmentation to cover the changed behavior [25] as well as prioritization or minimization to make its execution feasible [25, 26]. The assumption is also valid for other applications of differential software testing that aim to discover security vulnerabilities or to show robustness issues. They represent unexpected behavior that is challenging to avoid $[16,18,27]$, and there are usually no existing tests to discover them. Therefore, there is a need to generate these inputs. Furthermore, the proposed techniques in this work are designed to be effective with only one valid seed input that is used to initially execute the application under test. This increases the applicability of the presented techniques because they only make little assumptions about the availability of existing tests for the application.

Concept The concept of Hybrid DifFerential Software Testing (HyDIFF) is to combine powerful techniques to tackle the problem of test input generation to reveal behavioral differences in software. Differential software testing approaches a difficult problem because it generally requires to reason about multiple program executions. The existing work on differential analysis does not provide yet any hybrid differential software testing approach. Furthermore, the existing single approaches for a differential analysis have their own limitations. Therefore, this work proposes the usage of a hybrid approach, which combines random-based exploration (fuzzing) and systematic exploration (symbolic execution). The hybrid setup includes the exchange of interesting inputs between both single approaches that run in parallel. Both approaches can benefit from each other, and the overall analysis can explore a larger state space, while quickly generating results.

In order to illustrate this idea, Fig. 2 shows the overall concept. By running fuzzing and symbolic execution in parallel, both single techniques can perform their own exploration and can incorporate interesting inputs from the other component as well. This supports fuzzing to overcome narrow constraints in the program by importing inputs from symbolic execution, which is a whitebox technique that can analyze and solve these constraints with a constraint solver. Additionally, this hybrid concept also provides guidance for symbolic execution to focus on interesting program areas triggered by inputs from the fuzzing component. The term interesting depends on the specific type of the differential analysis. For example, in the context of regression analysis, an interesting input exposes a divergence between two program versions, while in the context of worst-case complexity analysis an interesting input maximizes the cost of the program execution. For a hybrid differential analysis, both single techniques need to be able to perform their own differential analysis. 
Fig. 2 Conceptual overview of the parallel hybrid exploration

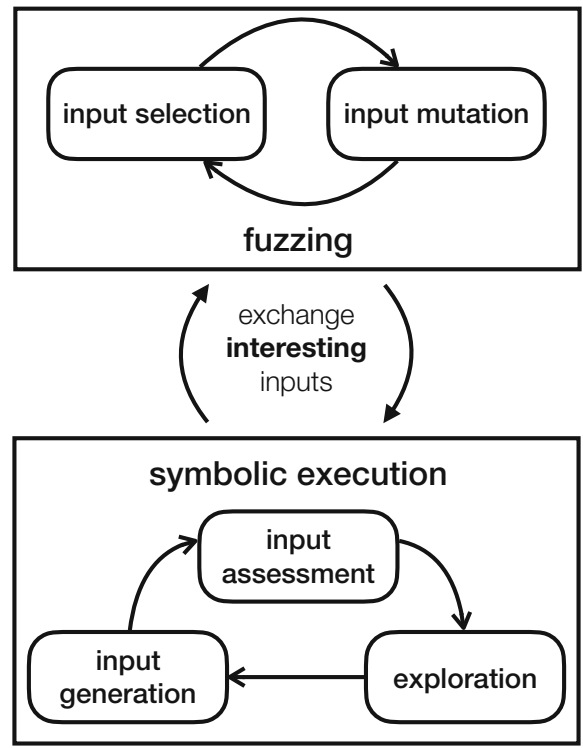

Differential Fuzzing (DF) Fuzzing as a representative of inexpensive, randombased exploration techniques [28] can generate a large number of inputs in a short time period due to its low overhead. Therefore, fuzzing is known for its input generation speed [24]. However, fuzzing is based on random operators and is usually implemented as blackbox or greybox technique. It lacks the knowledge about the program to go beyond complex constraints that guard deeper program behavior [24].

Similarly to standard fuzzing techniques, the proposed differential fuzzing process consists of a loop between input selection and input mutation (see the upper box in Fig. 2). The input mutation applies various mutation operators to generate new inputs. The input selection determines which mutated inputs are reported as interesting and which are kept for following evolutions. In order to perform a differential analysis, the input selection is driven by differential heuristics.

Differential Dynamic Symbolic Execution (DDSE) Existing approaches based on (dynamic) symbolic execution $[29,30]$ provide a systematic exploration of the state space, which can be guided by several heuristics. They have the full knowledge about the program, and hence, can unleash the full spectrum of program analysis techniques, for example, to reach low-probability branches. However, the scope of symbolic execution is usually limited to smaller programs because its systematic exploration encounters the path explosion problem and expensive path constraints solving [24]. Therefore, it does not scale to real-world applications.

The proposed differential (dynamic) symbolic execution consists of a loop between input assessment, exploration, and input generation (see the lower box in Fig. 2). The input assessment performs a concolic execution of concrete inputs, which includes the analysis of the executed branches based on differential heuristics. 
This analysis results in the identification and ranking of unexplored branches. The highest ranked, unexplored branch is used as starting point for some additional symbolic exploration. The resulting constraints of newly explored paths are extracted and used to generate new concrete inputs. These inputs are again assessed with concolic execution, which also reports interesting inputs for the fuzzing component.

\section{Differential Fuzzing}

Fuzzing Background Fuzzing [28] is a powerful technique to generate inputs that reveal errors (e.g., crashes) in programs. Recent fuzzing research efforts focus on optimizing the search process to find more crashes and cover more code [31, 32]. The metric to select new inputs from the mutated inputs for keeping them in the mutation corpus is only based on the ability to increase the code coverage. Leveraging a fuzzer for differential program analysis appears to be interesting due to the fact that behavioral differences might be triggered by unexpected inputs, which is exactly what fuzzing is made for. Nevertheless, for a differential program analysis the current fuzzing approaches need to be significantly extended. The fuzzer should not only generate inputs for crashes or increased code coverage but should specifically search for difference revealing inputs. In addition to coveragebased fuzzing, there are also efforts on directing fuzzing to specific program areas, for example, with AFLGo [33]. However, such approaches cannot be explicitly targeted to differential behavior. Nevertheless, such guiding capabilities are crucial because, for example, hitting the areas of changed code is a key ability to find regression bugs.

In general, a mutation-based fuzzer can be guided by three parameters: the seed inputs, the applied mutation operators, and the selection mechanism.

Guidance by Seed Inputs Guiding a fuzzer by its seed inputs requires existing inputs that already touch interesting areas of the program under test. Although in general an existing test suite can provide good seed inputs, it is a rather strong assumption that there are enough existing test inputs to sufficiently guide fuzzing. Additionally, a fuzzer will likely leave the areas touched by the seed inputs quite fast, based on its random mutation operators. A differential fuzzer should be able to make progress even without good seed inputs and at the same time it should have the possibility to incorporate new seed inputs during its fuzzing process.

Guidance by Mutation Operators Guiding a fuzzer with its mutation operators is already implemented to some extent in the state-of-the-art greybox fuzzers. For example AFL [34] implements mutation operators, which insert "known interesting integers" like 0,1 , or maximum values of data types like Integer. MAX_VALUE. Such mutation operators make sense when searching purely for crashes in programs, but might not be as efficient for a differential analysis. In differential analyses we need to guide the fuzzer first in interesting areas of the program, where actually a difference is occurring. 
Guidance by Selection Finally, the third option to guide the fuzzing process is the selection mechanism. In evolutionary algorithms, as they are used in fuzzing [31, $34]$, the mutant selection is the core guidance procedure. The selection procedure determines whether a mutant is kept in the mutation corpus and, hence, is reused for future mutations, or whether a mutant is eliminated. Therefore, a smart selection of mutants significantly helps to guide the fuzzer into interesting program behaviors. Typically, a fuzzer like AFL would identify inputs that produce program crashes, program hangs, or which cover new program branches because the goal is to find exactly these program behaviors. Inputs that produce crashes and hangs are moved into separate output folders and are no longer used for further mutations. Only the inputs that increase the coverage are kept in the mutation corpus. A differential fuzzer has slightly different needs: Inputs that lead to crashes and hangs should be still sorted out and not kept for further mutations. However, they should only be reported if they reveal behavioral differences. On the other hand, not only inputs that increase the branch coverage should be kept in the mutation corpus but also inputs that, for example, get closer to a change, show some output difference, or show some difference in its program exploration. Therefore, a differential fuzzer needs to be guided by a various set of differential metrics, like output difference, decision difference, cost difference, and patch distance. Additionally, a differential fuzzer should also keep inputs that increase the program coverage, to further guide it into unexplored areas.

Differential Fuzzing (DF) is defined as a method to identify behavioral differences with a guided mutational fuzzing approach.

Approach Overview In order to provide differential fuzzing, this work extends coverage-guided fuzzing by modifying the mutant selection mechanism (see Fig. 3). The presented approach uses several differential metrics like output difference, decision difference, cost difference, and patch distance to assess the behavioral properties of the mutated inputs. The focus is on coverage-guided, mutational fuzzing because the related work has shown that such a search-based fuzzing approach is highly effective. Furthermore, it does not make strong assumptions about the existing testing artifacts, for example, test suites or input grammars. Mutation-based fuzzing is built on a genetic algorithm, which belongs to the class of global search algorithms, known to be flexible, that is, being able to overcome local maxima, and to scale up well to larger problems [35]. However, note that the idea of differential fuzzing is not limited to this kind of fuzzing technique.

Figure 3 shows the overview of the proposed differential fuzzing technique. Similar to coverage-based, mutational fuzzing, it starts with some initial seed inputs (see step 1 in Fig. 3). It uses a queue (see step 2 in Fig. 3) to store the current fuzzing corpus. In order to generate new mutants, it first trims the inputs (see step 3) and afterwards applies several mutation operators on the inputs (see step 4). The main 
difference to standard greybox fuzzing is in the mutation selection mechanism (see step 5), which is specifically designed to select mutants that show new interesting behavioral properties. As shown in step 5 in Fig. 3, it takes an input and parses it to extract the various parameters of the application, which are then used to evaluate the input on multiple program executions. Finally, the various observations are compared and the differences are determined. Overall, differential fuzzing keeps inputs that show new interesting behaviors for future mutant generation (see step 6).

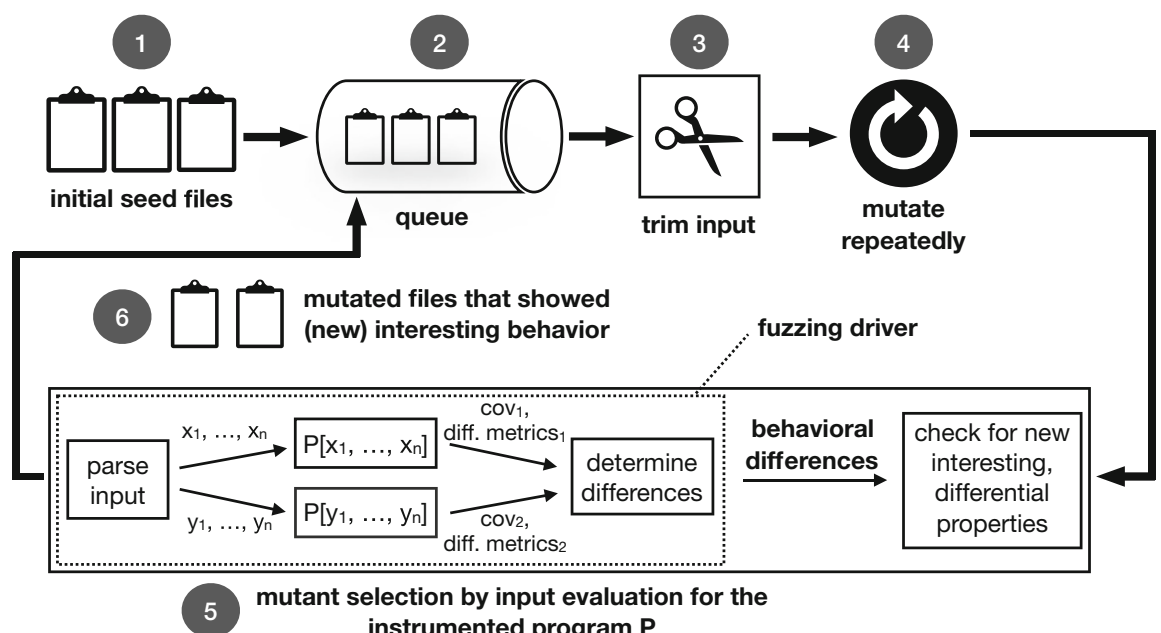

Fig. 3 Conceptual overview of differential fuzzing

\section{Differential Dynamic Symbolic Execution}

Symbolic Execution Background Symbolic execution [30] is well known for traversing the application in a systematic way. Under some assumption like that constraints can be solved in a reasonable time, or that third-party libraries calls can be analyzed or appropriate models are available, symbolic execution can efficiently generate test inputs to touch interesting program behavior. However, out-of-the-box symbolic execution has the limitation that it focuses on only one software version at once. A differential analysis, like regression analysis, is hence not possible with the standard symbolic execution approach.

In the last decade, a couple of approaches have been proposed to perform some sort of differential analysis with symbolic execution in the area of regression analysis [13, 14, 36, 37]. In summary, the recent advances provide the basis for a general differential testing approach, although none of them provide all necessary aspects. They either perform their analysis only on the new version, rely too much on concrete inputs for its guidance, or suffer from scalability issues, which makes it 
hard for a practical application. Moreover, all of them focus on regression analysis and not on a general differential analysis. For a scalable, general, and differential analysis, it needs a dynamic approach, to drive the exploration in interesting program areas. Furthermore, the differential analysis should be able to analyze multiple program versions at the same time to simplify constraints and prioritize paths early that show the best chances to reveal divergences. The analysis should allow the guidance by syntactic information about the program changes, so that paths can be pruned efficiently.

Differential Dynamic Symbolic Execution (DDSE) is defined as a systematic exploration of the program's input space, characterized by symbolic values, which is specifically focused and guided on generating inputs that reveal behavioral differences.

Approach Overview Shadow symbolic execution [13] proposes the exploration of change-annotated programs, which represents an elegant way of combining multiple versions or allowing multiple differential behaviors in one execution. As described in [6], shadow symbolic execution [13] might miss important divergences due to its strong dependence on concrete inputs. The proposed approach in [6], complete shadow symbolic execution, explores the usage of the four-way forking idea in standard symbolic execution, without having any concrete inputs to drive the exploration. While such an approach does not miss divergences, as long as the program can be explored exhaustively, it obviously comes with its own scalability issues. Therefore, the proposed solution for differential dynamic symbolic execution (DDSE) allows the usage of concrete inputs to drive the exploration, but still uses a complete four-way forking approach to detect all divergences in the search space. Consequently, DDSE is driven by differential expressions introduced by changeannotations inside the program and is further guided by differential metrics like the cost difference and the patch distance. In a hybrid differential analysis setup, DDSE is receptive for guidance based on concrete inputs from the fuzzing component.

Figure 4 shows the overview of the proposed differential dynamic symbolic execution approach, consisting of five phases: (1) import of inputs, (2) input assessment, (3) exploration, (4) input generation, and (5) export of inputs. Note that all symbolic execution variants presented in Fig. 4, that is, concolic execution, trieguided symbolic execution, and bounded symbolic execution, support the execution of a change-annotated program. All together, denoted with the dashed area in Fig. 4, form the so-called differential dynamic symbolic execution.

Central Data Structure: Trie The central data structure in this dynamic symbolic execution is a so-called trie, which has been adapted from Yang et al. [37]. A trie represents a subset of the symbolic execution tree, where nodes represent the choices during symbolic execution that include symbolic variables. Therefore, a trie is a simplified variant of a symbolic execution tree, where only the components 
are included, which are interesting for the analysis and which are necessary to replay specific paths in the tree. In this approach it used to store the current state of the analysis (see step 2 in Fig. 4) and to select a promising point to continue the exploration (see step 3 in Fig. 4).

(1) Input Import The process starts with importing initial seed inputs (see step 1 in Fig. 4). Note: The term importing inputs refers to the fact that such an import can not only be performed in the beginning of the analysis but also periodically throughout the whole process. This functionality is crucial for the synchronization with another technique in a hybrid setup.

(2) Input Assessment The given inputs are executed concolically, that is, the symbolic execution follows only the path of the concrete values but collects all symbolic information (i.e., the value mapping and the path constraint) along this path (see step 2 in Fig. 4). The execution is mapped to the simplified symbolic execution tree called trie. After the concolic execution of the given inputs, each node, which has unexplored branches, represents a potential entry point for further exploration. The nodes are analyzed and ranked with the defined heuristics, which is followed by the selection of the most promising node.

(3) Exploration The idea behind the expanded exploration step (see step 3 in Fig. 4) is to discover new, interesting parts of the state space. In order to reach the actual symbolic state at the selected node it starts with a trie-guided symbolic execution and switches to a bounded symbolic execution as soon as it hits the selected node.

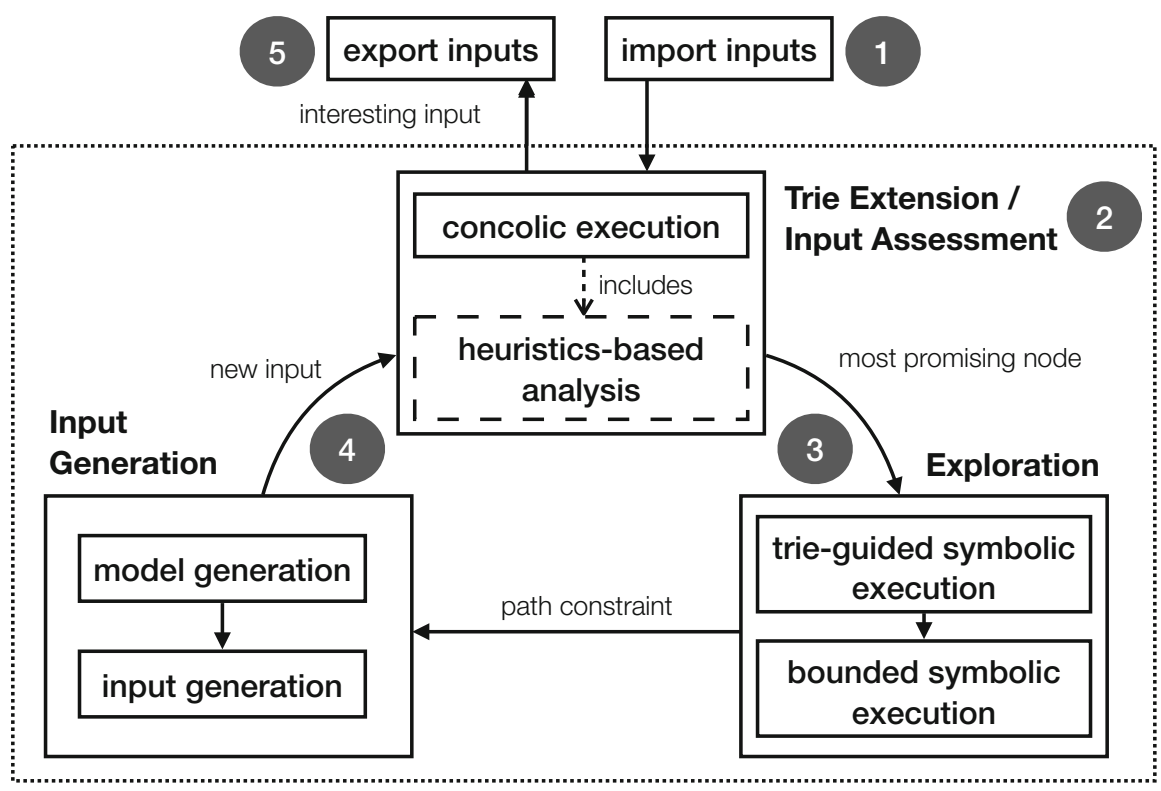

Fig. 4 Conceptual overview of dynamic, heuristic-driven symbolic execution; based on [4] 
Trie-guided means that the symbolic execution simply follows the choices stored in the trie without any invocation of a constraint solver. This step is very efficient and builds the symbolic state. As soon as hitting the selected node, the execution switches to a bounded symbolic execution mode, which will perform an exhaustive symbolic execution up to a predefined bound. The exploration step results in a sequence of satisfiable path constraints.

(4) Input Generation In step 4 in Fig. 4 an SMT solver is leveraged to generate a model for each path constraint. Afterwards, these models are used to construct inputs. The input generation is application-specific since the path constraints and their models have no information about the actual input formats and requirements. Note that the inputs have been generated based on the exploration of a promising node determined by heuristics. This means that after the generation the inputs need to be assessed for their actual usefulness for the current analysis. Therefore, they are executed concolically (see step 2 in Fig. 4), and the trie is extended.

Altogether, the steps 2, 3, and 4 form an analysis loop (see dashed area in Fig. 4). The loop can be paused for the import of new inputs (e.g., in the hybrid setup), it can be stopped by a user-specified bound, or it is finished after the complete (i.e., exhaustive) exploration.

(5) Input Export As soon as a generated input is assessed as interesting, that is, it shows some new behavior interesting for the current analysis, it is reported for the export (see step 5 in Fig. 4). In a hybrid setup the exported inputs are made available for the other technique; in a single analysis setup, the exported inputs represent the output of the analysis.

\section{General Framework for Hybrid Differential Software Testing}

The general framework HYDIFF is instantiated as the combination of the hybrid concept (see Sect.2) with the concrete solutions for its components differential fuzzing (see Sect.3) and differential dynamic symbolic execution (see Sect.4). HyDIFF's overview is presented in Fig. 5.

Inputs The upper part of Fig. 5 shows the input of HyDIFF, which takes one or two program version(s) (used for fuzzing) and the change-annotated program (used for symbolic execution). Additionally, the approach expects one or more seed input files to drive the exploration, which are shared by both components.

Collaborations Between Components The middle part of Fig. 5 shows the two components and their workflow. In contrast to the existing related work on hybrid analysis [24, 38], the proposed approach executes fuzzing and symbolic execution in parallel and not in a sequential order. The intuition is that both techniques are highly effective on their own but benefit from some guidance into certain areas 


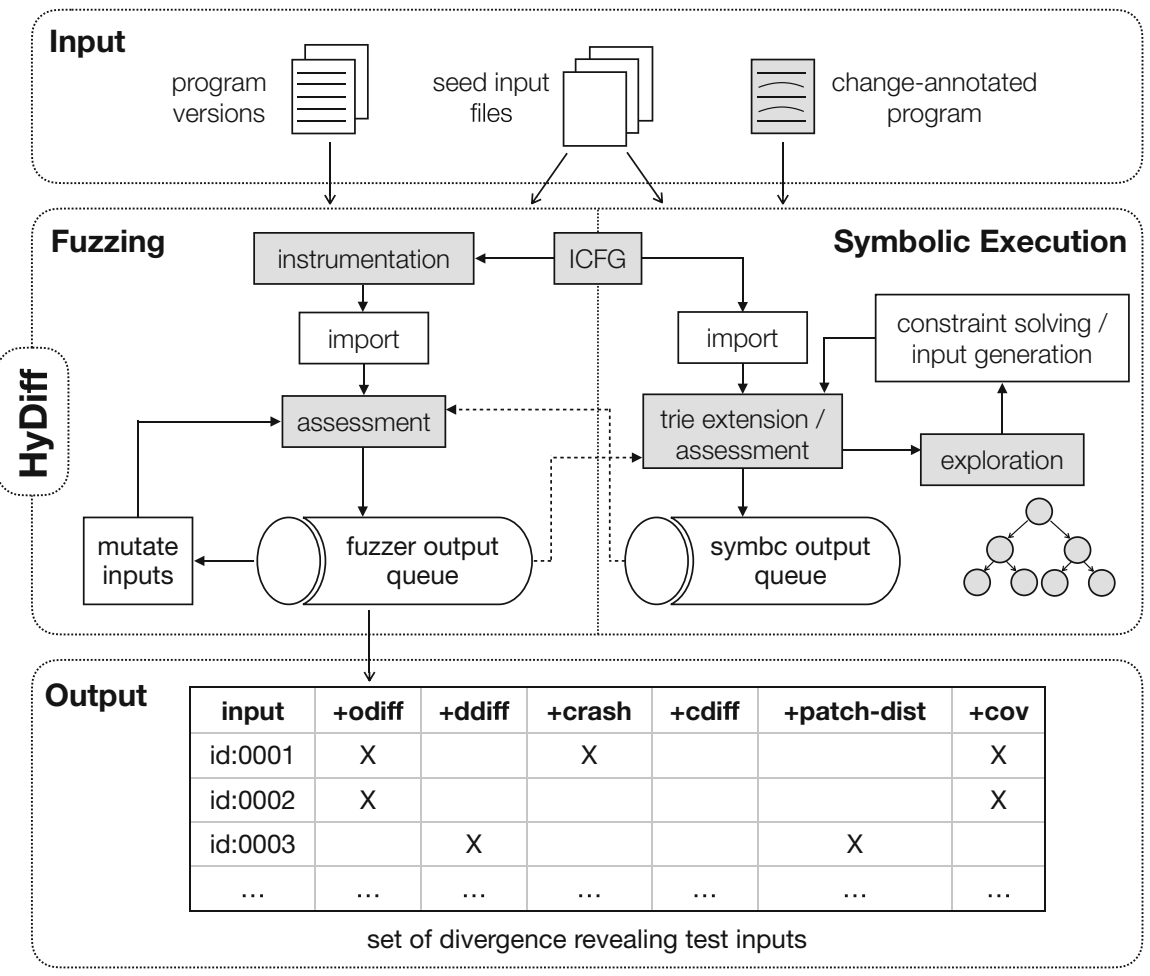

Fig. 5 Overview of HYDIFF's workflow [7]

of the search space. Conceptually this is performed by importing the inputs from the other's output queue (see the arrows from the output queues to the assessment nodes in both sides in the middle of Fig. 5). Additionally, both components use the information from the inter-procedural control flow graph (ICFG) to drive/prune their exploration, which is, for example, relevant for regression testing. DF leverages the ICFG to calculate the distance values, which are used to determine whether inputs get closer to the changed location(s), and hence, to guide the DF toward the modification(s). DDSE uses it to prune paths that cannot reach any changed area.

Outputs The lower part of Fig. 5 shows the expected output of the hybrid analysis, which is a list of generated inputs and their characteristics in terms of differential behavior. They are classified according to the differential metrics. 


\section{Applications}

The main research interest in hybrid differential software testing is to expose software bugs related to differential behavior. Finding such bugs is essential to improve the software quality in general. The contributions by this work aim at providing efficient and effective techniques to contribute to this research interest. In particular, they aim at supporting software developers in creating reliable and secure software, and hence, also facilitate the main research idea in software engineering, namely to support software development with methods, techniques, and tools. Therefore, HYDIFF needs to be evaluated on how its two components proceed, whether their combination can amplify the exploration, and finally, how effective HYDIFF is in general for differential software testing. These aspects are evaluated based on a quantitative analysis with experiments and benchmarks in the presented application areas of differential program analysis. The following sections present exemplary insights and summaries from this evaluation for each application area.

Note that in addition to differential fuzzing (DF) the evaluation also reports results for parallel differential fuzzing (PDF), which represents a variant of DF with two parallel running fuzzer instances. PDF mitigates the parallel nature of HYDIFF in the evaluation and provides a more fair comparison. For DDSE it is hard to provide a similar parallel variant because parallel symbolic execution is its own research problem. Simply running two symbolic execution instances in parallel would not provide any benefit due to its deterministic behavior. The evaluation therefore also included a DDSE variant with double time budget; however, there was no significant improvement with regard to the identified differences.

\subsection{Regression Analysis (A1)}

Regression analysis is one of the main applications of differential program analysis where the goal is to identify behavioral differences between two successive software versions. The most meaningful semantic different behaviors are differences in the actual output of an application like the result of a calculation or another output message. Unintended behavioral differences are called regression errors. Regression analysis is also one of the "most extensively researched areas in [software] testing" [11] and is therefore the major focus of HYDIFF.

Example Listing 1 shows a change-annotated program, combining two versions of the program calculate. This program is an artificial example, which shows the strengths and drawbacks of fuzzing and symbolic execution. It processes two integer inputs, $x$ and $y$, and calculates a division based on these two values. The large switch statement with cases from 0 to 250 (lines 4-7) is a challenge for symbolic execution because there are a lot of branches to explore. In this example it is especially problematic because none of them can be pruned because all of them can reach the changed condition in line 18 , and the interesting part is at the 
Listing 1 Example program for regression testing with HyDIFF [7]

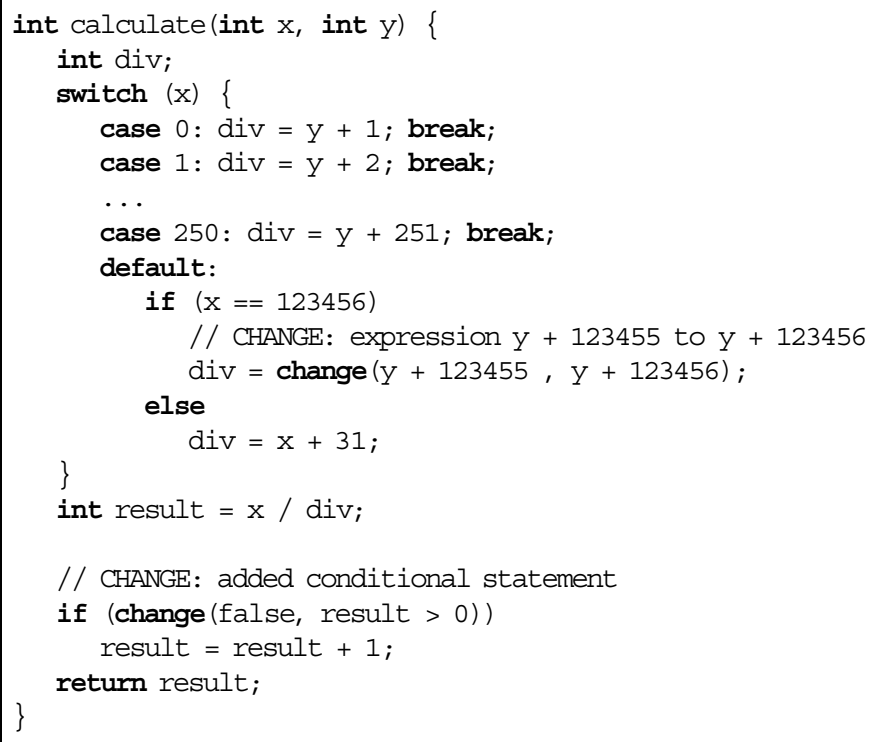

end of the switch statement, which will be reached late in the exploration (when having a deterministic exploration order). In the default case of the switch statement (line 8), there is a check for the value 123456 representing a magic value, which guards the first change in line 11. There the developer changed the right-hand-side expression from $y+123455$ to $y+123456$, which fixed a division-by-zero error for $y=-123455$, but introduced another crash for $y=-123456$. In contrary to symbolic execution, fuzzing is expected to traverse the program quite fast, but it will have problems with handling the magic number. In line 19, the developer added a conditional statement result $=$ result +1 if result $>0$. This influences the output for all positive results. However, it does not directly fix or introduce any crash.

To further illustrate the challenges of each individual component, the following paragraph first discusses the results for running both components in isolation and afterwards together in the hybrid setup. The differential fuzzing component finds its first output difference after $5.07( \pm 0.99) \mathrm{sec}$ (where the \pm value denotes the $95 \%$ confidence interval over 30 runs). In total it finds 1.37 ( \pm 0.17$)$ output differences and $1.00( \pm 0.00)$ decision differences. The new crash is not found within the time bound of $10 \mathrm{~min}$. Therefore, fuzzing is very fast in finding an output difference (less than $5 \mathrm{~s}$ ), but the narrow constraint at the end is difficult to reach for fuzzing: $(\mathrm{x}=123456 \& \mathrm{y}=-123456)$. In contrast, the differential dynamic symbolic execution component finds its first output difference after $135.27( \pm 0.66)$ s. In total, it finds $35.10( \pm 1.10)$ output differences and $2.00( \pm 0.00)$ decision differences. So it reveals much more output differences than fuzzing within the given time bound. 
In fact, the DDSE component can traverse all paths in 5 min. In contrast to fuzzing it also finds the new crash, after $135.80( \pm 0.64)$ s. Nonetheless, symbolic execution needs relatively long to find its first output difference. In the hybrid setup, the differential fuzzing and symbolic execution components are started with the same seed input. Both run their analysis in parallel and exchange inputs that are deemed interesting according to the divergence metrics after a prespecified time bound. The experimental results are as follows: first output difference after $4.73( \pm 0.78) \mathrm{s}$, in total $35.13( \pm 1.04)$ output differences and $2.00( \pm 0.00)$ decision differences. The hybrid technique finds the new crash already after $14.43( \pm 0.30) \mathrm{s}$. Figure 6 shows the temporal development of the results for the three techniques. Although DDSE and HYDIFF come to similar conclusions after $10 \mathrm{~min}$, HYDIFF is significantly faster in finding the first output differences (as well as the crash). DF is fast in generating first results, but cannot achieve the same numbers as DDSE and HYDIFF within the $10 \mathrm{~min}$ time bound.
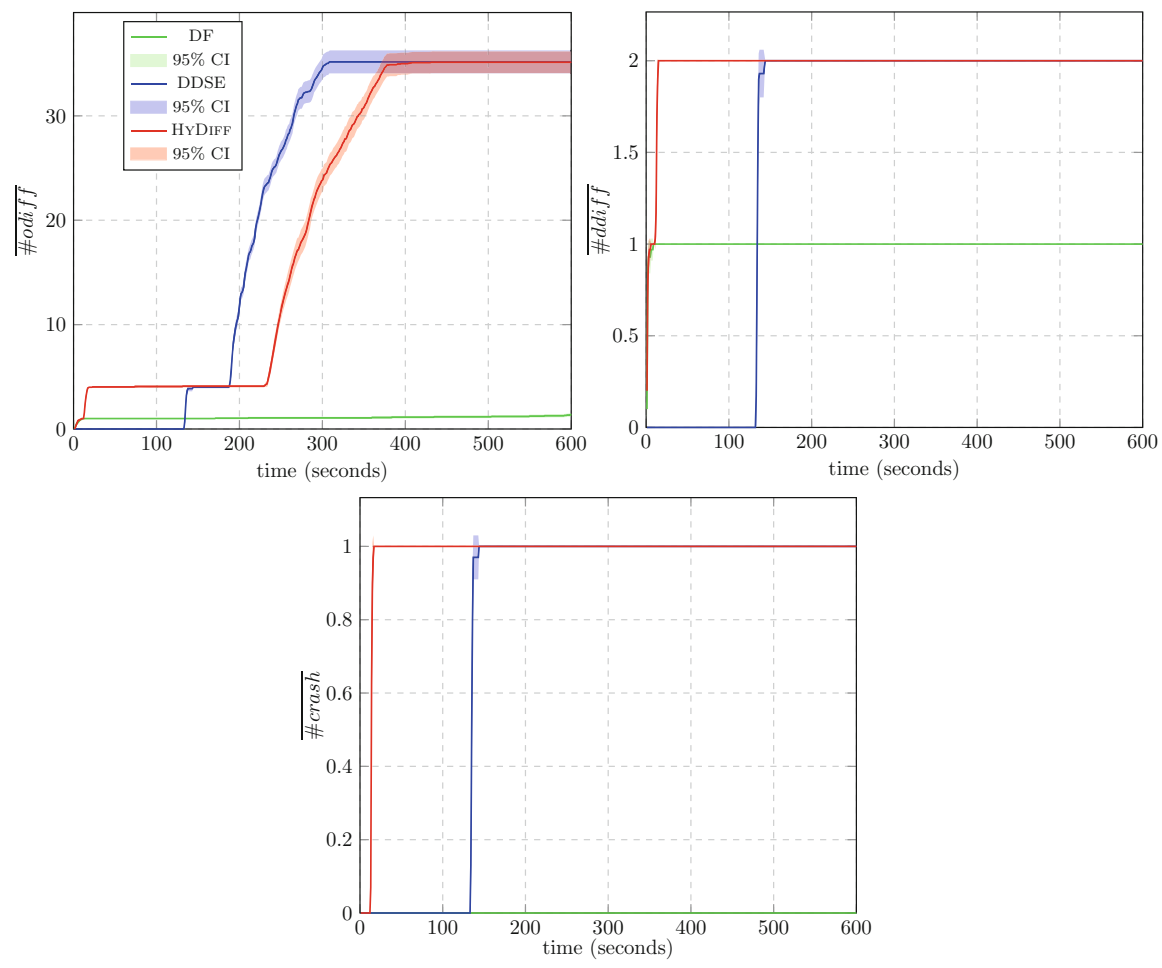

Fig. 6 Results for DF, DDSE, and HYDIFF on hybrid approach sample (lines and bands show averages and $95 \%$ confidence intervals across 30 repetitions)

The hybrid approach detects the regression bug more than nine times faster than DDSE component in isolation. The DF component (in isolation) times out after 
10 min without detecting the regression bug. The hybrid setup can leverage the strengths of both techniques, so that it can get into many more paths by using symbolic execution and quickly find its first output difference by using fuzzing.

Summary of Evaluation For the evaluation of the application regression analysis the subjects are taken from multiple versions of the Traffic Collision Avoidance System (TCAS) [39] as well as subjects from the Defects4J benchmark [40]. Additionally, HYDIFF is evaluated on subjects from Apache Commons CLI library [41]. The conducted experiments indicate that the symbolic execution component of HYDIFF can greatly benefit from the combination with fuzzing and vice versa. The performance of differential fuzzing can be improved by running it in a parallel setup, but still it cannot classify all subjects correctly. In contrast, HYDIFF does classify all subjects correctly, that is, it identifies for all subjects output differences, for which they actually exist. Furthermore, the overall evaluation shows that HYDIFF still outperforms DDSE, DF, and PDF for the presented subjects.

\subsection{Worst-Case Complexity Analysis (A2)}

Worst-case complexity analysis (WCA) searches for worst-case execution paths, which represent a serious threat to the system under test. The main objective is not to identify the theoretical complexity of an algorithm but to identify inputs that trigger a worst-case execution behavior. Similar to the next application sidechannel analysis, it is a highly relevant application in the security area because such a characterization of an algorithm's complexity can help, for example, to identify worst-case complexity vulnerabilities [16]. The current need for such techniques is also recognized by the US Defense Advanced Research Projects Agency (DARPA), which recently organized the Space/Time Analysis for Cybersecurity (STAC) program [42] that supported the development of new analysis techniques to identify algorithmic complexity and side-channel vulnerabilities. Due to the simpler nature of the worst-case complexity analysis (compared to the other application scenarios), this approach is quite straightforward because it does not require the reasoning about multiple execution paths at the same time. The basic goal is to maximize the observed execution cost.

Example Listings 2 shows an implementation of the sorting algorithm Insertion Sort taken from JDK 1.5, for which the worst-case execution behavior (in terms of the runtime) is known: $N^{2}$, where $N$ denotes the length of the input array. The worst-case would be triggered by a reverse-ordered array.

Table 1 shows the results for applying DF, PDF, DDSE, and HYDIFF on the presented Insertion Sort subject. The experiments have been executed for $1 \mathrm{~h}$ and repeated for 30 times. The columns in this table show the average maximum cost obtained within the given time bound $(\bar{c})$, the maximum cost value over all runs $\left(c_{\max }\right)$, and the time in seconds until the first cost improvement with regard to 
Listing 2 Sample program for WCA: Insertion Sort

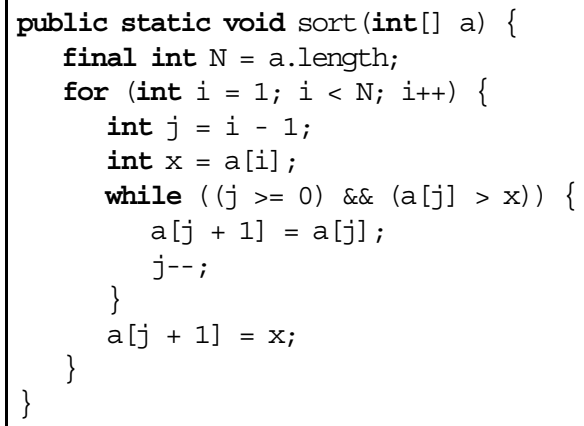

Table 1 Results for the Insertion Sort with $N=64(t=3600 \mathrm{~s}=60 \mathrm{~min}, 30$ runs). The execution cost $c$ is measured as the number of executed JAVA bytecode instructions

\begin{tabular}{l|l|l|l}
\hline Technique & $\bar{c}$ & $c_{\max }$ & $t: c^{-}>0$ \\
\hline DF & $9048.40( \pm 85.51)$ & 9567 & $5.70( \pm 0.16)$ \\
\hline PDF & $9355.03( \pm 41.53)$ & 9571 & $5.10( \pm 0.11)$ \\
\hline DDSE & $1157.00( \pm 00.00)$ & 1157 & $2.13( \pm 0.15)$ \\
\hline HYDIFF & $9693.77( \pm 42.44)$ & 9923 & $2.93( \pm 0.16)$ \\
\hline
\end{tabular}

the cost value of the initial input $(\bar{t}: c>0)$, which had a cost value of 509 bytecode instructions. The numbers in Table 1 show that HyDIFF can generate inputs with significantly higher costs. However, within the $1 \mathrm{~h}$ time bound, none of the techniques has been able to identify the worst-case input. The maximum cost value generated by HYDIFF was 9923 and the actual worst-case cost value would be 10,526 for a totally reverse-ordered array with $N=64$. Nonetheless, the input by HYDIFF with 9923 gets very close to this worst-case (see Listing 3). Therefore, HYDIFF achieves on average a slowdown of ca. $19.04 \times$, that is, that the identified cost value as $19.04 \times$ more expensive than the cost value of the initial input.

Listing 3 HYDIFF's worst-performing input for Insertion Sort $N=64(t=60 \mathrm{~min})$

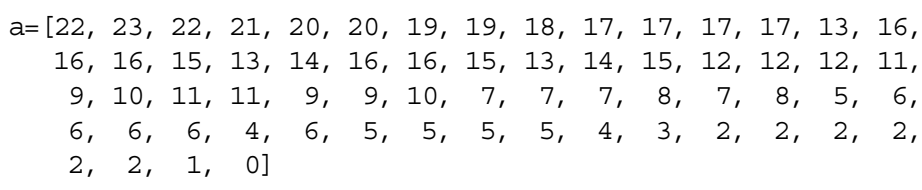

Parallel differential fuzzing (PDF) takes the second position with a slowdown of $18.38 \times$ followed by single differential fuzzing (DF) with a slowdown of $17.78 \times$. Far behind is differential dynamic symbolic execution (DDSE) with a slowdown of $2.27 \times$, which cannot achieve high cost values. However, with regard to the time 
to the first cost improvement, DDSE is the best followed by HYDIFF. Note that these differences are quite small and also the fuzzing techniques show very similar behavior.

More interesting is the comparison of the techniques over the analysis time like shown in Fig. 7. During the first 2 min DF, PDF, and HYDIFF perform very similar, but afterwards HYDIFF can break away and can generate an average cost value of 9000 executed bytecode instructions within $8.2 \mathrm{~min}$, for which PDF needs 27.5 and DF $54.6 \mathrm{~min}$.

Fig. 7 Results for DF, PDF, DDSE, and HYDIFF on the Insertion Sort Example with $N=64$ (lines and bands show averages and $95 \%$ confidence intervals across 30 repetitions)

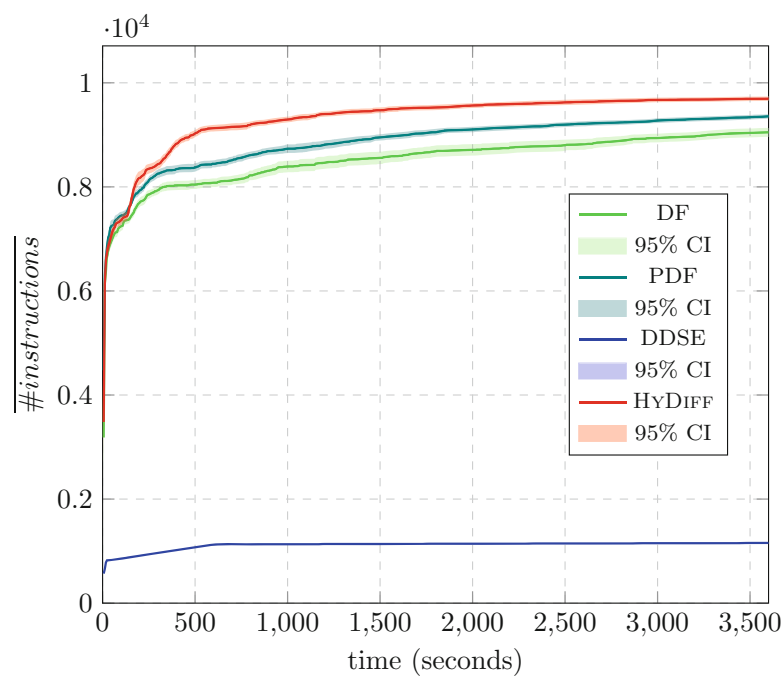

Summary of Evaluation For the evaluation of application worst-case complexity analysis, the subjects are chosen based on the evaluation of the approach SLOWFuZz [16], since it is the most related work in this context. The data set includes textbook algorithms like Insertion Sort and regular expression matching from the JAVA JDK. It also includes algorithms from the STAC program [42] and real-world applications, for example, APACHE COMMONS COMPRESS [43].

HYDIFF successfully combines the strengths of DDSE and DF. Symbolic execution helps HYDIFF to quickly make progress and fuzzing supports by continuously improving the score. Therefore, in the majority of the cases, HYDIFF is as good as its components or even outperforms them. In particular, HYDIFF's strength is to quickly generate a high cost value, for which the other techniques take quite long. 


\subsection{Side-Channel Analysis (A3)}

Side-channel analysis searches for information leakages that are caused by diverging cost-behaviors within the same application. As already mentioned for A2, side-channel analysis is highly relevant with regard to security and is in the focus of recent research projects. The popular Meltdown [44] and Spectre [45] side-channel attacks also gave it some publicity outside the research community. Side-channel analysis is difficult because it requires the reasoning about multiple execution paths at the same time and additionally involves the handling of cost behaviors.

The key idea for the implementation of side-channel analysis with HYDIFF is to use the idea of self-composition [46] and consider two execution paths, which both are initialized with the same public input but different secret inputs. The goal is to maximize the cost difference $(\delta)$ between these two execution paths. The higher the cost difference can be identified, the more severe is a side-channel vulnerability. This idea is described by the following formula:

$$
\underset{\text { pub,sec } 1, \text { sec }_{2}}{\operatorname{maximize}} \delta=\mid c\left(P \llbracket p u b, \sec _{1} \rrbracket\right)-c\left(P \llbracket p u b, \text { sec }_{2} \rrbracket\right) \mid
$$

In this formula $p u b$ denotes the public value, and $\sec _{1}$ and $s e c_{2}$ denote the two secret values. $P \llbracket p u b, \sec _{1} \rrbracket$ denotes the execution of program $P$ with the public value $p u b$, and the secret value $\sec _{1} . c(P \llbracket . . \rrbracket)$ denotes the cost measurement of the execution of program $P . \delta$ denotes the cost difference of both program executions.

For HYDIFF's fuzzing component this means to fuzz three values: the public value and two secret values. Note that this approach naturally extends to tuples of values. The most important metric to detect the side-channel vulnerability is the cost difference between these two executions, but the fuzzer will also collect the information about decision differences and output differences. They are still important metrics to drive the fuzzing process, although they cannot directly measure the severity of a side-channel vulnerability. For HYDIFF's symbolic execution component, the variation in the secret input can be realized by the usage of change-annotations:

$$
\text { secret }=\text { change }\left(\text { secret }_{1}, \text { secret }_{2}\right)
$$

The differential dynamic symbolic execution does also require three inputs (one public and two secret values), but does combine the two secret values in one changeannotated expression. This means that for symbolic execution there is only one program execution necessary. Since this change-annotation happens directly in the driver, the program itself does not contain any change-annotation, and hence, the patch distance metric is not relevant for side-channel analysis. Also the control-flow information cannot help to prune any path because the differential expression is introduced straight in the beginning. The primary goal of the symbolic execution in the hybrid setup is to support the fuzzing component by solving complex branching conditions, which are infeasible for fuzzing. 
Example To illustrate the analysis approach, consider the unsafe password comparison algorithm in Listing 4. The algorithm takes two arrays as parameters, one array for the (public) user input pub and the other for the stored (secret) password sec. It starts with comparing the length of both arrays and will return false if both lengths do not match. As long as both arrays have the same length, the algorithm continues with comparing the passwords byte by byte. As soon as there is a mismatch, the algorithm will return false, and only if all byte values match, the algorithm will finally return true. Therefore, this unsafe algorithm has two early-returns, in lines 2 and 4, which are the reason for the vulnerability. In order to avoid the timing sidechannel, it would be necessary to iterate over the complete public input without having such early returns.

Listing 4 Side-channel analysis example: an unsafe password checking algorithm

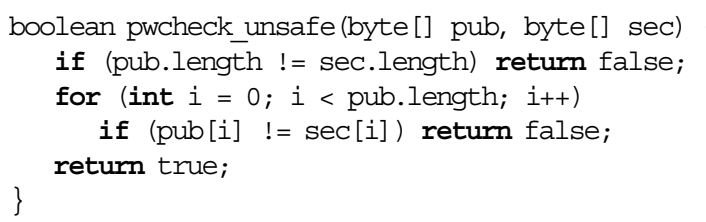

Table 2 shows the experiment results for applying DF, PDF, DDSE, and HYDIFF on the password checking example. The experiments have been executed with a time bound of $5 \mathrm{~min}$ and have been repeated 30 times. The number of executed bytecode instructions are used as cost metric, which is an alternative to measure the real runtime of the algorithm. Other processes running on the machine are expected to influence the actual real-time measurement, and hence, counting the executed bytecode instruction is a more robust metric.

Table 2 Results for the unsafe password checking example ( $t=300 \mathrm{~s}=5 \mathrm{~min}, 30$ runs)

\begin{tabular}{l|l|l|c}
\hline Technique & $\bar{\delta}$ & $\delta_{\max }$ & $\bar{t}: \delta>0$ \\
\hline Differential fuzzing (DF) & $34.30( \pm 3.11)$ & 47 & $4.20( \pm 1.53)$ \\
\hline Parallel differential fuzzing (PDF) & $40.93( \pm 1.84)$ & 47 & $2.33( \pm 0.63)$ \\
\hline Differential dynamic symbolic execution (DDSE) & $47.00( \pm 0.00)$ & 47 & $13.27( \pm 0.24)$ \\
\hline HYDIFF & $47.00( \pm 0.00)$ & 47 & $4.43( \pm 1.00)$ \\
\hline
\end{tabular}

Listing 5 Input for maximum cost difference after $5 \mathrm{~min}$

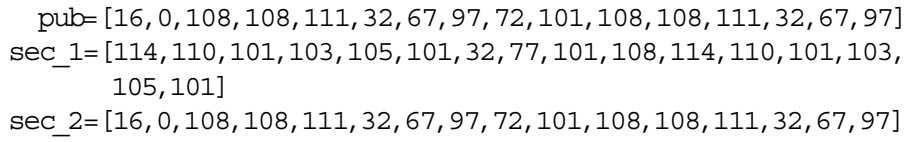

For this experiment, the maximum input size was set to 16 bytes, which allows a maximum cost difference of 47 bytecode instructions. Listing 5 shows an input, which triggers the maximum cost difference. 
Fig. 8 Results for DF, PDF, DDSE, and HYDIFF on the password checking example (lines and bands show averages and $95 \%$ confidence intervals across 30 repetitions)

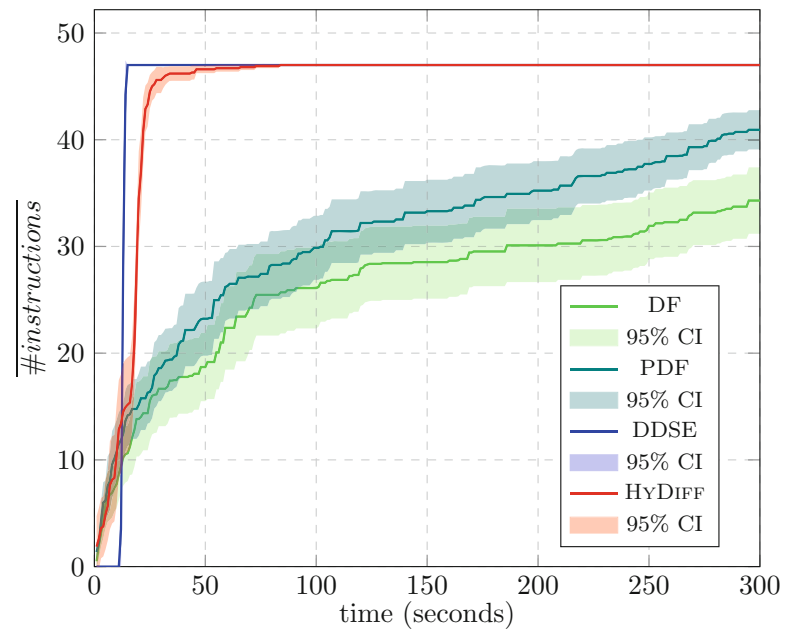

All of the four experiment setups (DF, PDF, DDSE, and HYDIFF) have been able to reach the maximum value at least once (see $\delta_{\max }$ column in Table 2 ). The values in the table also show that DF and PDF cannot reliably generate this maximum value within the time bound of $5 \mathrm{~min}$. However, the fuzzing techniques show a better performance in identifying the first input for an improved $\delta$ value (see column $\bar{t}: \delta>0$ ). Figure 8 shows the temporal development. DF, PDF, and HyDifF perform quite similar in the beginning, whereas DDSE takes longer to generate an interesting input. After approximately $13 \mathrm{~s}$, DDSE jumps almost directly to the actual maximum cost difference. HYDIFF follows quickly, whereas DF and PDF take longer to get to this maximum value.

Summary of Evaluation The evaluation of application side-channel analysis includes subjects from the evaluation of two state-of-the-art static analysis tools BLAZER [17] and THEMIS [18]. Additionally, the evaluation includes more subjects from the STAC program [42] and the modular exponentiation known from the RSA encryption technique [47].

Although HYDIFF shows some peaks in the evaluation, it is usually not much better than the naive combination of the results from both components. Nevertheless, HYDIFF represents a well-balanced combination. As it combines both techniques, it can identify a cost difference very fast and is able to assess the severity of side-channel vulnerabilities very well because it can quickly identify large $\delta$ values. For all subjects HYDIFF and its components can efficiently detect sidechannel vulnerabilities. Furthermore, the comparison between differential fuzzing and BLAZER/THEMIS shows that HYDIFF can keep up with state-of-the-art static analysis tools for the detection of side-channel vulnerabilities. Additionally, HYDIFF's fuzzing component has been used to reveal multiple new vulnerabilities, which later got fixed by the developers (as presented in [2]). 


\subsection{Robustness Analysis of Neural Networks (A4)}

Robustness analysis of neural networks is a relatively novel application of differential program analysis where the goal is to identify vulnerabilities in neural networks with regard to their robustness. It requires reasoning about multiple network executions, which makes it very expensive, and hence, serves as a stress testing application of HYDIFF and its components. HYDIFF is specifically used to find adversarial inputs for an image classification network. Since HyDIFF performs the analysis of JAVA bytecode, the first step is to rewrite a given neural network model into a JAVA program [48]. Similarly to the side-channel analysis, the idea for the differential analysis is to allow changes in the input and observe differences in the network's behavior. More precisely, the proposed analysis changes up to $x \%$ of the pixels in the input image and checks whether there can be any difference in the output.

Some preliminary experiments have shown that with HYDIFF in its default setup (i.e., differential fuzzing and differential dynamic symbolic execution start at the same time), both components do not synchronize with each other because they are busy with their own analysis due to the expensive program execution. Therefore, the execution setup for the neural network analysis is different: the experiments start with differential symbolic execution for $10 \mathrm{~min}$. After this time bound the differential fuzzing component is started with the already generated inputs by the differential symbolic execution component as additional seed inputs and both component run in parallel for the remaining time. The $10 \mathrm{~min}$ delay provides sufficient time to the DDSE component to generate a first interesting input.

Example Listing 6 shows an extract of the JAVA program, which represents the transformed neural network model for the experiments for this case study. The program takes a double array as input, which represents a normalized $28 \times 28$ grayscale image. The image is expected to include a handwritten digit and the network has been trained to recognize such digits. Each compartment of this program denotes a layer of the neural network. The final layer shows the final classification of the hand-written digits into the numbers $0-9$.

The implemented analysis tries to identify two images that differ only up to a given threshold and that are differently classified by the neural network. In order to illustrate the effect of this approach, consider Fig. 9. It shows two inputs that have been generated by differential fuzzing after $1375 \mathrm{~s}$. The image on the left side is classified as the digit 6 and the image on the right side is classified as the digit 5 . The generated images are not necessarily representative as adversarial inputs as they do not fulfill the assumption of the neural network, namely that it expects handwritten digits. Due to the analysis implementation, the search process can change the complete image, and so, these images look randomly generated. Note that this case study is used as stress testing for HYDIFF and its components. Therefore, it is fair enough to identify images that differ slightly and lead to different classifications in order to assess the robustness of the neural network. 
Listing 6 Extract of the transformed neural network model as JAVA program

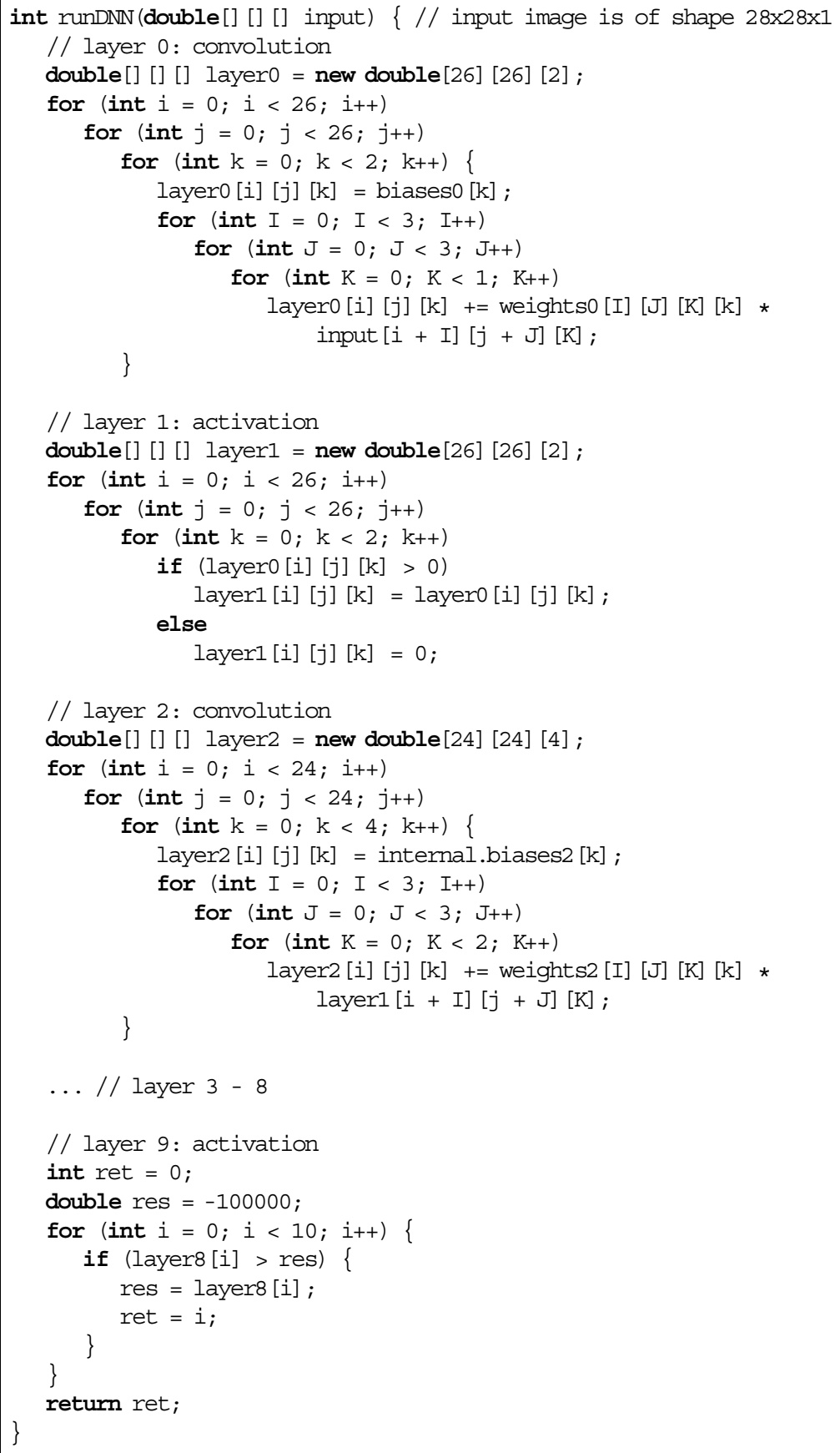


However, it is also possible to update the analysis to perform a more realistic scenario. If the original input is kept and the process searches only the pixel locations and values, then the result can be used as adversarial inputs. This analysis would be much harder because there are not so many ways to change an existing image. Figure 10 shows an exemplary result of performing such an analysis with differential fuzzing. The left side shows the original image that is classified as 6 . The right side shows an image with $50 \%$ changed pixel that is classified as 8 . In the experiment, differential fuzzing needed more than $60 \mathrm{~h}$ to generate the adversarial input.

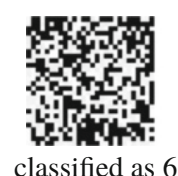

classified as 6

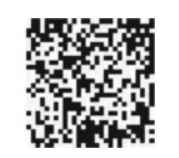

classified as 5

Fig. 9 Inputs identified by DF after $1375 \mathrm{~s}$ by fuzzing two images that differ in up to $1 \%$ of the pixels. The learned model classifies the left image as a 6 , while it classifies the right image as a 5 . The images differ only in 7 pixels

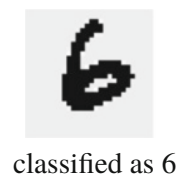

Fig. 10 Adversarial changes identified by DF after $60.89 \mathrm{~h}$ by fuzzing up to $50 \%$ pixel changes for a fixed image. The learned model classifies the left (original) image as a 6 , while it classifies the right image as an 8 . The images differ in 314 pixels

Summary of Evaluation The results for this experimental setup show the different benefits of the two different approaches (fuzzing and symbolic execution) and why it is important to combine them! HYDIFF leverages its differential symbolic execution component to quickly generate a first output difference and further leverages differential fuzzing to identify even more output differences. HYDIFF does not only combine the results of both components, but the components can benefit from each other's inputs to further improve the outcome. Overall, the evaluation for the neural network subjects has shown that all three techniques (DF, DDSE, and their hybrid combination HYDIFF) can be used to find adversarial inputs for neural networks. Although this scenario shows the limitation of all approaches, output differences have been generated. 


\section{Conclusion and Future Work}

This work contributes the concept of hybrid differential software testing (HYDIFF) as a combination of differential fuzzing (DF) and differential dynamic symbolic execution (DDSE). HYDIFF's fuzzing component employs a search-based differential exploration implemented by a genetic algorithm. Its benefit is the inexpensive generation of inputs as well as the generation of unexpected inputs due to the random mutation strategies. HYDIFF's symbolic execution component performs a systematic exploration guided by several differential heuristics. Because it can incorporate concrete inputs at runtime, it also can be driven by the inputs of the fuzzing component. It further can overcome specific constraints due to its constraintsolving capabilities. This supports fuzzing, which might not reach deep program behaviors due to its random nature. Overall, HYDIFF strengthens the presented differential fuzzing technique by combining it with the heuristic-based, systematic exploration in symbolic execution. As combination this supports a wide spectrum of differential testing applications and contributes a generally usable testing technique.

For evaluation purpose, this work showed its applicability on several application scenarios: regression analysis, worst-case complexity analysis, side-channel analysis, and robustness analysis of neural networks. This multifaceted evaluation shows that HYDIFF can be applied in numerous testing disciplines, and so, contributes to the overall research interest of software testing. Additionally, the application of fuzzing for side-channel vulnerability detection already had a direct impact outside of the research community, namely on the development of more fuzzing tools with SIDEFUZZ [49]. HyDIFF complements the existing work on hybrid testing and provides a baseline for future research. In summary, the contributions made by this work (especially the technical abilities to reveal behavioral differences) represent an important step in the direction of better (i.e., more secure and more reliable) software, and hence, support the overall goal of software engineering.

The conducted research also has revealed interesting future research directions. First of all, the application section mentioned the parallel variant HyDIFF's fuzzing component. It would be interesting to further analyze its performance, which could lead to corresponding parallel fuzzing guidelines, and furthermore, it would be interesting to develop a parallel symbolic execution variant. Additionally, the research around HYDIFF aims at the generation of test inputs, which can be continued to perform actual debugging and repair of the identified errors and vulnerabilities. Therefore, an interesting future work could be the automated repair in the areas of software evolution and security vulnerabilities and their combination with the techniques proposed in this work.

Acknowledgments I want to thank my family and friends for supporting me during my $\mathrm{PhD}$. My great appreciation goes to all my co-authors and my particular gratitude to Lars Grunske and Corina Păsăreanu for their great support. 


\section{References}

1. Noller, Y.: Hybrid differential software testing. Ph.D. Thesis, Humboldt-Universität zu Berlin, Mathematisch-Naturwissenschaftliche Fakultät (2020). https://doi.org/10.18452/21968

2. Nilizadeh, S., Noller, Y., Păsăreanu, C.S.: Diffuzz: differential fuzzing for side-channel analysis. In: Proceedings of the 41st International Conference on Software Engineering, ICSE '19, pp. 176-187. IEEE Press, Piscataway (2019). https://doi.org/10.1109/ICSE.2019.00034

3. Noller, Y.: Differential program analysis with fuzzing and symbolic execution. In: Proceedings of the 33rd ACM/IEEE International Conference on Automated Software Engineering, ASE 2018, pp. 944-947. ACM, New York (2018). https://doi.org/10.1145/3238147.3241537

4. Noller, Y., Kersten, R., Păsăreanu, C.S.: Badger: complexity analysis with fuzzing and symbolic execution. In: Proceedings of the 27th ACM SIGSOFT International Symposium on Software Testing and Analysis, ISSTA 2018, pp. 322-332. ACM, New York (2018). https:// doi.org/10.1145/3213846.3213868

5. Noller, Y., Nguyen, H.L., Tang, M., Kehrer, T.: Shadow symbolic execution with java pathfinder. SIGSOFT Softw. Eng. Notes 42(4), 1-5 (2018). https://doi.org/10.1145/3149485. 3149492

6. Noller, Y., Nguyen, H.L., Tang, M., Kehrer, T., Grunske, L.: Complete shadow symbolic execution with java pathfinder. SIGSOFT Softw. Eng. Notes 44(4), 15-16 (2019). https://doi. org/10.1145/3364452.33644558

7. Noller, Y., Păsăreanu, C.S., Böhme, M., Sun, Y., Nguyen, H.L., Grunske, L.: Hydiff: Hybrid differential software analysis. In: Will appear in: Proceedings of the 42nd International Conference on Software Engineering, ICSE '20 (2020)

8. ISO/IEC/IEEE International Standard-Systems and software engineering-Vocabulary. ISO/IEC/IEEE 24765:2017(E), pp. 1-541 (2017). https://doi.org/10.1109/IEEESTD.2017. 8016712

9. DeRemer, F., Kron, H.H.: Programming-in-the-large versus programming-in-the-small. IEEE Trans. Softw. Eng. 2(2), 80-86 (1976). https://doi.org/10.1109/TSE.1976.233534

10. Vliet, H.V.: Software Engineering: Principles and Practice, 3rd edn. Wiley, London (2008)

11. Orso, A., Rothermel, G.: Software testing: A research travelogue (2000-2014). In: Proceedings of the on Future of Software Engineering, FOSE 2014, pp. 117-132. Association for Computing Machinery, New York (2014). https://doi.org/10.1145/2593882.2593885

12. Fraser, G., Arcuri, A.: A large-scale evaluation of automated unit test generation using evosuite. ACM Trans. Softw. Eng. Methodol. 24(2) (2014). https://doi.org/10.1145/2685612

13. Palikareva, H., Kuchta, T., Cadar, C.: Shadow of a doubt: testing for divergences between software versions. In: 2016 IEEE/ACM 38th International Conference on Software Engineering (ICSE), pp. 1181-1192 (2016). https://doi.org/10.1145/2884781.2884845

14. Person, S., Dwyer, M.B., Elbaum, S., Păsăreanu, C.S.: Differential symbolic execution. In: Proceedings of the 16th ACM SIGSOFT International Symposium on Foundations of Software Engineering, SIGSOFT '08/FSE-16, pp. 226-237. ACM, New York (2008). https://doi.org/10. $1145 / 1453101.1453131$

15. Luckow, K., Kersten, R., Păsăreanu, C.S.: Symbolic complexity analysis using contextpreserving histories. In: 2017 IEEE International Conference on Software Testing, Verification and Validation (ICST), pp. 58-68 (2017). https://doi.org/10.1109/ICST.2017.13

16. Petsios, T., Zhao, J., Keromytis, A.D., Jana, S.: Slowfuzz: Automated domain-independent detection of algorithmic complexity vulnerabilities. In: Proceedings of the 2017 ACM SIGSAC Conference on Computer and Communications Security, CCS '17, pp. 2155-2168. ACM, New York (2017). https://doi.org/10.1145/3133956.3134073

17. Antonopoulos, T., Gazzillo, P., Hicks, M., Koskinen, E., Terauchi, T., Wei, S.: Decomposition instead of self-composition for proving the absence of timing channels. SIGPLAN Not. 52(6), 362-375 (2017). https://doi.org/10.1145/3140587.3062378

18. Chen, J., Feng, Y., Dillig, I.: Precise detection of side-channel vulnerabilities using quantitative Cartesian Hoare logic. In: Proceedings of the 2017 ACM SIGSAC Conference on Computer 
and Communications Security, CCS '17, pp. 875-890. Association for Computing Machinery, New York (2017). https://doi.org/10.1145/3133956.3134058

19. Păsăreanu, C.S., Phan, Q.S., Malacaria, P.: Multi-run side-channel analysis using symbolic execution and Max-SMT. In: 2016 IEEE 29th Computer Security Foundations Symposium (CSF), pp. 387-400 (2016). https://doi.org/10.1109/CSF.2016.34

20. Sun, Y., Wu, M., Ruan, W., Huang, X., Kwiatkowska, M., Kroening, D.: Concolic testing for deep neural networks. In: Proceedings of the 33rd ACM/IEEE International Conference on Automated Software Engineering, ASE 2018, pp. 109-119. Association for Computing Machinery, New York (2018). https://doi.org/10.1145/3238147.3238172

21. Tramer, F., Boneh, D.: Adversarial training and robustness for multiple perturbations. In: Wallach, H., Larochelle, H., Beygelzimer, A., d'Alché-Buc, F., Fox, E., Garnett, R. (eds.) Advances in Neural Information Processing Systems, vol. 32, pp. 5858-5868. Curran Associates, Red Hook (2019)

22. Goodfellow, I.J., Shlens, J., Szegedy, C.: Explaining and harnessing adversarial examples (2014). arXiv preprint arXiv:1412.6572

23. Cha, S.K., Avgerinos, T., Rebert, A., Brumley, D.: Unleashing mayhem on binary code. In: 2012 IEEE Symposium on Security and Privacy, pp. 380-394 (2012). https://doi.org/10.1109/ SP.2012.31

24. Stephens, N., Grosen, J., Salls, C., Dutcher, A., Wang, R., Corbetta, J., Shoshitaishvili, Y., Kruegel, C., Vigna, G.: Driller: augmenting fuzzing through selective symbolic execution. In: 23nd Annual Network and Distributed System Security Symposium, NDSS 2016, San Diego, California, USA, February 21-24, 2016 (2016). https://doi.org/10.14722/ndss.2016.23368

25. Harrold, M.J.: Testing evolving software. J. Syst. Softw. 47(2), 173-181 (1999). https://doi. org/10.1016/S0164-1212(99)00037-0

26. Yoo, S., Harman, M.: Regression testing minimization, selection and prioritization: a survey. Softw. Testing Verif. Reliab. 22(2), 67-120 (2012). https://doi.org/10.1002/stvr.430

27. Brennan, T., Saha, S., Bultan, T., Păsăreanu, C.S.: Symbolic path cost analysis for side-channel detection. In: Proceedings of the 27th ACM SIGSOFT International Symposium on Software Testing and Analysis, ISSTA 2018, pp. 27-37. ACM, New York (2018). https://doi.org/10. $1145 / 3213846.3213867$

28. Miller, B.P., Fredriksen, L., So, B.: An empirical study of the reliability of Unix utilities. Commun. ACM 33(12), 32-44 (1990). https://doi.org/10.1145/96267.96279

29. Godefroid, P., Klarlund, N., Sen, K.: Dart: Directed automated random testing. In: Proceedings of the 2005 ACM SIGPLAN Conference on Programming Language Design and Implementation, PLDI '05, pp. 213-223. Association for Computing Machinery, New York (2005). https:// doi.org/10.1145/1065010.1065036

30. King, J.C.: Symbolic execution and program testing. Commun. ACM 19(7), 385-394 (1976). https://doi.org/10.1145/360248.360252

31. Böhme, M., Pham, V.T., Roychoudhury, A.: Coverage-based greybox fuzzing as Markov chain. In: Proceedings of the 2016 ACM SIGSAC Conference on Computer and Communications Security, CCS '16, pp. 1032-1043. ACM, New York (2016). https://doi.org/10.1145/2976749. 2978428

32. Pham, V.T., Böhme, M., Santosa, A.E., Căciulescu, A.R., Roychoudhury, A.: Smart greybox fuzzing. IEEE Trans. Softw. Eng. 1-17 (2019)

33. Böhme, M., Pham, V.T., Nguyen, M.D., Roychoudhury, A.: Directed greybox fuzzing. In: Proceedings of the 2017 ACM SIGSAC Conference on Computer and Communications Security, CCS '17, pp. 2329-2344. ACM, New York (2017). https://doi.org/10.1145/3133956. 3134020

34. Website: American fuzzy lop (AFL) - a security-oriented fuzzer (2014). http://lcamtuf. coredump.cx/afl/

35. Zeller, A., Gopinath, R., Böhme, M., Fraser, G., Holler, C.: The fuzzing book. In: The Fuzzing Book. Saarland University (2019). https://www.fuzzingbook.org/

36. Person, S., Yang, G., Rungta, N., Khurshid, S.: Directed incremental symbolic execution. In: Proceedings of the 32Nd ACM SIGPLAN Conference on Programming Language Design and 
Implementation, PLDI '11, pp. 504-515. ACM, New York (2011). https://doi.org/10.1145/ 1993498.1993558

37. Yang, G., Păsăreanu, C.S., Khurshid, S.: Memoized symbolic execution. In: Proceedings of the 2012 International Symposium on Software Testing and Analysis, ISSTA 2012, pp. 144-154. ACM, New York (2012). https://doi.org/10.1145/2338965.2336771

38. Majumdar, R., Sen, K.: Hybrid concolic testing. In: 29th International Conference on Software Engineering (ICSE'07), pp. 416-426. IEEE Computer Society, Los Alamitos (2007). https:// doi.org/10.1109/ICSE.2007.41

39. Website: Software-artifact infrastructure repository (2019). http://sir.unl.edu

40. Just, R., Jalali, D., Ernst, M.D.: Defects4j: A database of existing faults to enable controlled testing studies for java programs. In: Proceedings of the 2014 International Symposium on Software Testing and Analysis, ISSTA 2014, pp. 437-440. ACM, New York (2014). https:// doi.org/10.1145/2610384.2628055

41. Website: Commons CLI (2019). https://commons.apache.org/proper/commons-cli/

42. Website: DARPA's Space/Time Analysis for Cybersecurity (STAC) program (2015). https:// www.darpa.mil/program/space-time-analysis-for-cybersecurity

43. Website: Debian bug report log 800564—php5: trivial hash complexity DoS attack (2015). https://bugs.debian.org/cgi-bin/bugreport.cgi?bug=800564

44. Lipp, M., Schwarz, M., Gruss, D., Prescher, T., Haas, W., Fogh, A., Horn, J., Mangard, S., Kocher, P., Genkin, D., Yarom, Y., Hamburg, M.: Meltdown: reading kernel memory from user space. In: 27th USENIX Security Symposium (USENIX Security 18), pp. 973-990. USENIX Association, Baltimore (2018)

45. Kocher, P., Horn, J., Fogh, A., Genkin, D., Gruss, D., Haas, W., Hamburg, M., Lipp, M., Mangard, S., Prescher, T., Schwarz, M., Yarom, Y.: Spectre attacks: exploiting speculative execution. In: 2019 IEEE Symposium on Security and Privacy (SP), pp. 1-19 (2019). https:// doi.org/10.1109/SP.2019.00002

46. Barthe, G., D'Argenio, P.R., Rezk, T.: Secure information flow by self-composition. In: Proceedings. 17th IEEE Computer Security Foundations Workshop, 2004, pp. 100-114 (2004). https://doi.org/10.1109/CSFW.2004.1310735

47. Kocher, P.C.: Timing attacks on implementations of Diffie-Hellman, RSA, DSS, and other systems. In: Koblitz, N. (ed.) Advances in Cryptology—CRYPTO '96, pp. 104-113. Springer, Berlin, Heidelberg (1996)

48. Website: Deep learning test toolset (2020). https://github.com/theyoucheng/DLTT

49. Website: SideFuzz: Fuzzing for side-channel vulnerabilities (2018). https://github.com/phayes/ sidefuzz

Open Access This chapter is licensed under the terms of the Creative Commons Attribution 4.0 International License (http://creativecommons.org/licenses/by/4.0/), which permits use, sharing, adaptation, distribution and reproduction in any medium or format, as long as you give appropriate credit to the original author(s) and the source, provide a link to the Creative Commons license and indicate if changes were made.

The images or other third party material in this chapter are included in the chapter's Creative Commons license, unless indicated otherwise in a credit line to the material. If material is not included in the chapter's Creative Commons license and your intended use is not permitted by statutory regulation or exceeds the permitted use, you will need to obtain permission directly from the copyright holder. 